\title{
Estrategias para interrumpir trampas de riesgo en Barrios Altos. Caso de estudio Quinta Baselli
}

\author{
Vanesa Olazábal Mendoza \\ Universidad Nacional Federico Villarreal, Perú \\ Recibido: 30 de junio del 2017 / Aprobado: 5 de septiembre del 2017 \\ doi: 10.26439/limaq2018.n004.2258
}

\begin{abstract}
Barrios Altos conserva en sus calles el relato de la historia de Lima, evidenciado en la cantidad de edificios de carácter monumental que posee. Sin embargo, el paso del tiempo ha transformado esta zona en una trampa de riesgo latente ante la expectativa de intereses privados que pretenden desresidencializarla y, en consecuencia, provocar la pérdida de memoria urbana, así como reducir las dinámicas sociales propias y culturalmente tradicionales del lugar. Partiendo de ello, este artículo expone las trampas de riesgo identificadas en Barrios Altos y los mecanismos participativos a escala barrial que se enfrentan a ellas. Asimismo, se presentan los resultados de la intervención independiente para la reducción de riesgos cotidianos, denominada Plan Piloto para la Recuperación de Quintas, ejecutada por el equipo técnico del Colectivo Más Ciudad con el financiamiento del Proyecto cLima Sin Riesgo de University College London.
\end{abstract}

patrimonio, Centro Histórico, renovación urbana, participación

ciudadana, resiliencia, riesgos cotidianos

\section{Strategies to stop risk traps in Barrios Altos. Quinta Baselli case study}

Barrios Altos preserves on its streets the history of Lima, evidenced by its several monumental buildings. Nevertheless, through the years, it has become an urban risk trap, due to the expectation of private interests seeking to move off the traditional citizens and, consequently, stimulate the loss of urban memory, and decrease the traditional social and cultural dynamics of the area. Based on this situation, the present article shows the risk traps identified in Barrios Altos and the neighborhood mechanisms facing them. In addition, the results of an independent intervention for reducing daily risks, named Plan Piloto para la Recuperación de Quintas, executed by the technical team of Colectivo Más Ciudad and financed by the Proyecto cLima Sin Riesgo of the University College London, are presented.

heritage, Historic Center, urban renovation, citizen participation, resilience, urban risk traps 


\section{INTRODUCCIÓN}

El Centro Histórico de Lima (CHL), declarado en 1991 Patrimonio de la Humanidad ${ }^{1}$ por la Unesco, rescata los valores culturales e históricos encerrados en un sector de su territorio, que es un testimonio de la transformación social y espacial ocurrida en el país desde el siglo XVI hasta la actualidad, la cual se evidencia en la estructura física que mantiene. El CHL tiene una extensión de $1022,81 \mathrm{Ha}$, de las cuales $68 \%$ pertenecen al distrito de Cercado de Lima, $28 \%$ al distrito del Rímac y 0,4 \% a otros distritos. El área declarada Patrimonio Cultural de la Humanidad es solo el $23 \%$ del total del CHL (Programa Municipal para la Recuperación del Centro Histórico de Lima [Prolima], 2014).

El territorio de Barrios Altos, ubicado al este del CHL, ha tenido una morfología variable desde sus orígenes prehispánicos y tras la imposición de la traza española del Damero de Pizarro en 1535. Su época de esplendor se dio tras la guerra del Pacífico, de 1890 a 1940, periodo en el que se levantaron importantes casonas solariegas. Después de ese lapso comenzó su decadencia, iniciada con el terremoto de 1940, que dañó muchas edificaciones de la zona y produjo la emigración de los residentes a distritos como San Isidro, Miraflores y Barranco. Por otro lado, el aumento de la densidad poblacional, debido al proceso migratorio del interior del país hacia la capital en la década de 1950, generó condiciones de hacinamiento, deterioro de infraestructura de vivienda y reducción de la calidad de vida en Barrios Altos (Municipalidad Metropolitana de Lima [MML], 1999).

Con la ruptura de la continuidad de la traza urbana debido a la construcción de la avenida Abancay en 1952, este sector se terminó por convertir en un espacio residual, a pesar de su céntrica localización para acceder a servicios metropolitanos. Hasta la fecha, Barrios Altos no ha sufrido grandes transformaciones que mejoren su situación; aún posee limitaciones de interconectividad, vivienda, espacios públicos, dotación de servicios básicos, equipamiento, entre otros.

1 Se entiende como centro histórico un conjunto urbano de carácter irrepetible en el que van marcando su huella los distintos momentos de la vida de un pueblo para formar la base en la que se asientan sus señas de identidad y su memoria social (Carta de Veracruz, 1992, p. 1). 

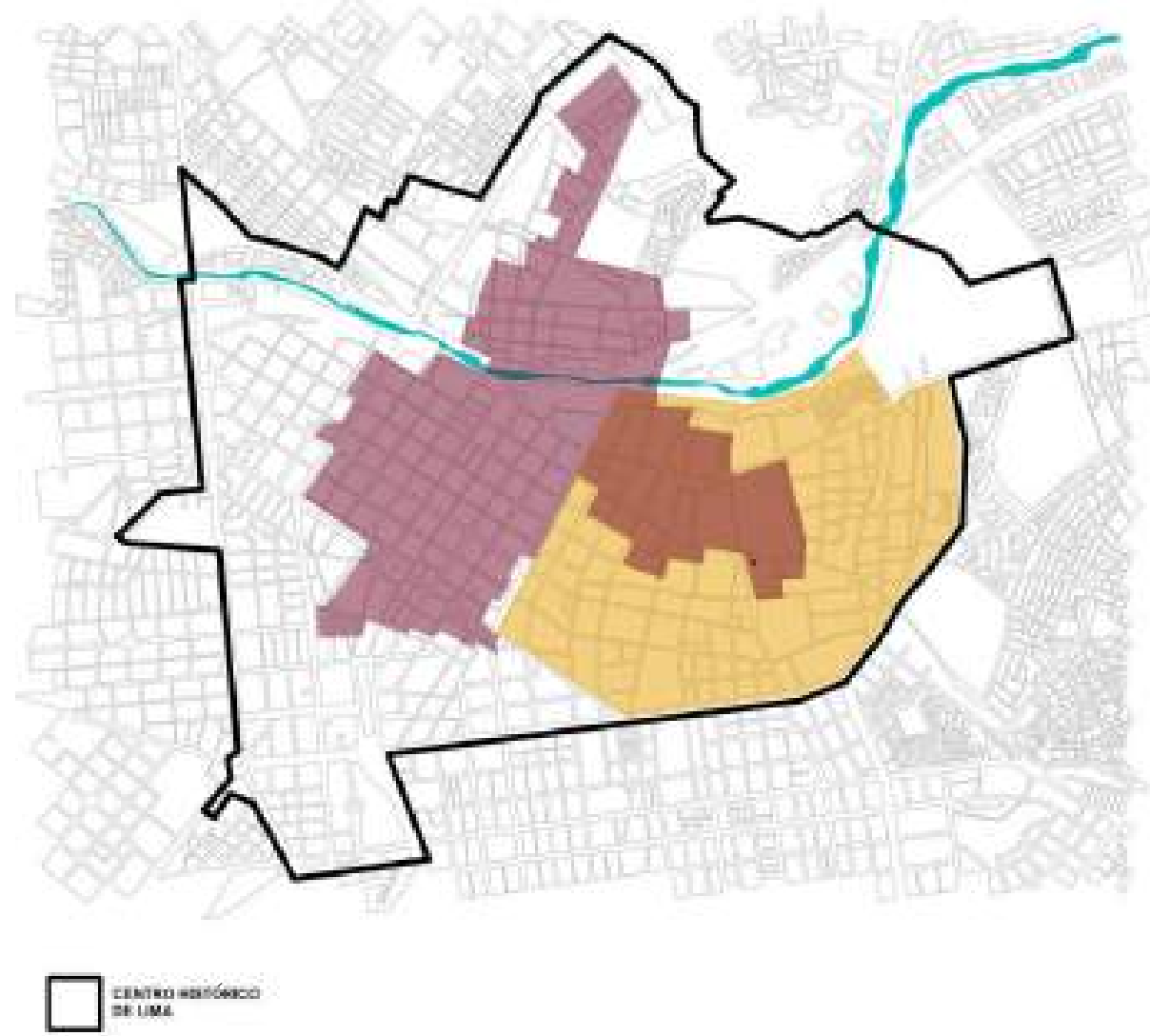

MTruceso contak De CA

Themans dechated

now Unesco

IASPCS n.tos

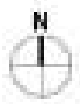

Maspos netos

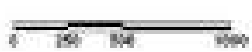

Figura 1

Mapa del Centro Histórico de Lima

Elaboración: Iván Rojas Villafuerte 
Ante la actual realidad de Barrios Altos surgen una serie de preguntas: ¿qué son trampas de riesgo urbano? ¿Está Barrios Altos expuesto a trampas de riesgo cotidiano? ¿Qué retos diarios enfrentan los residentes de este lugar? ¿Qué estrategias pueden servir para encarar los riesgos cotidianos? ¿Qué acciones toman sus actores?

\section{CONTEXTO GENERAL: IDENTIFICANDO TRAMPAS DE RIESGO COTIDIANO EN BARRIOS ALTOS}

Según lo define el proyecto cLima sin Riesgo ${ }^{2}$, las trampas de riesgo cotidiano refieren a un proceso cíclico de peligros recurrentes que se acumulan por causas ocasionadas por amenazas y vulnerabilidades no atendidas en grupos sociales definidos por un contexto urbano. En dicho contexto se procederá a identificar las trampas de riesgo cotidiano encontradas en Barrios Altos.

\section{Deterioro urbano-ambiental}

La traza urbana irregular de Barrios Altos, junto con la ocupación progresiva no regulada de viviendas, ha generado la ocupación habitacional del $50 \%$ del territorio y ha dejado solo el $10 \%$ destinado a áreas libres. Esto ha ocasionado una densidad poblacional de $557 \mathrm{hab} / \mathrm{Ha}$, alta para el índice edificatorio del sector, que es de dos a cuatro pisos (Centro de Promoción Urbana, 2009).

La sobreocupación del territorio trae consigo problemas de hacinamiento que aumentan la insalubridad, relacionados con la proliferación de enfermedades contagiosas y la contaminación atmosférica por la mala gestión de residuos. Por otro lado, las áreas verdes del CHL ascienden a 1,33 $\mathrm{m}^{2} / \mathrm{hab}$. (Prolima, 2014), cifra que se encuentra lejos del estándar de $9 \mathrm{~m}^{2} /$ hab. de áreas verdes referido frecuentemente. Todo ello genera que Barrios Altos cuente con apenas el $20 \%$ de los requerimientos mínimos en torno a salubridad y habitabilidad (Centro de Promoción Urbana, 2009).

2 cLima sin Riesgo es un proyecto de investigación-acción de temática social y ambiental que se encuentra en la búsqueda de ciudades justas y resilientes, a cargo del Development Planning Unit del University College London. 
Por otro lado, los escasos espacios públicos del sector no satisfacen las actividades demandadas por la población joven. Los niños no poseen lugares seguros de juego psicomotor, mientras que los jóvenes no encuentran áreas para realizar actividades deportivas. Todo ello trae consigo problemas sociales, aumenta el estrés tanto del usuario como el de su familia y fomenta la inseguridad ciudadana al no ocupar a su población joven en actividades recreativas.

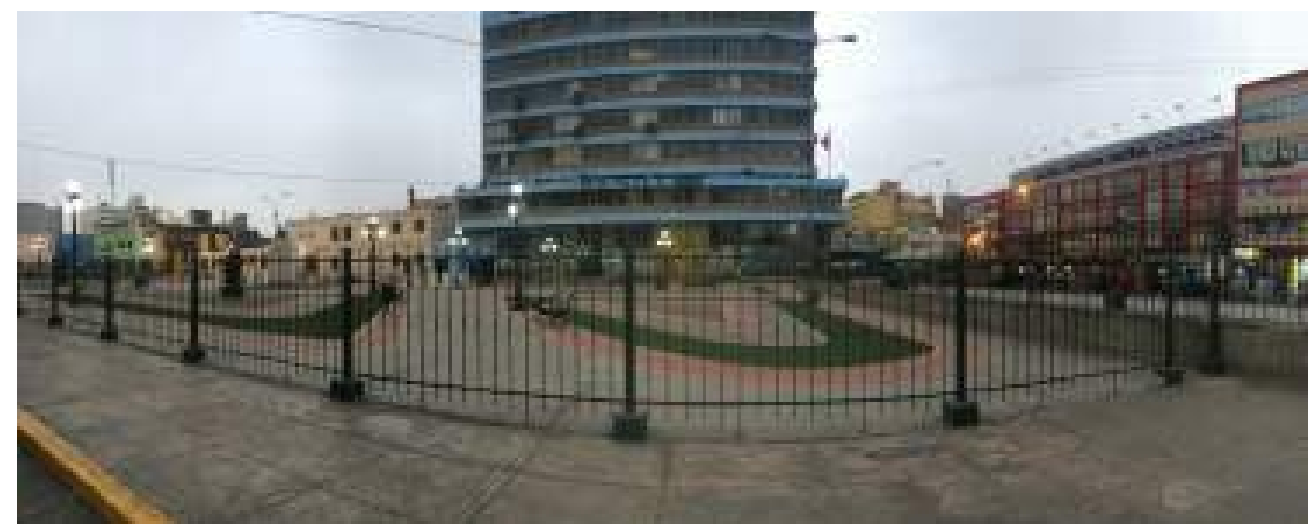

Figura 2

Plaza Castañeta

Fotografía: Vanesa Olazábal Mendoza ${ }^{3}$

\section{Déficit de calidad de vivienda}

Las tipologías de vivienda en Barrios Altos son en su mayor parte casonas, quintas y callejones, los que representan el $80 \%$ de los edificios residenciales (Indeci, 2001). En términos de materiales constructivos, los más usados son la quincha y el adobe, presentes en el $36 \%$ de las edificaciones en el CHL (Prolima, 2014).

3 Todas las fotografías pertenecen a la autora del artículo. 
Por la naturaleza de los materiales constructivos, su temporalidad y la falta de mantenimiento generalizado, se identifica el debilitamiento estructural como recurrente problemática: $61 \%$ de las construcciones en Barrios Altos presentan humedad, $52 \%$ rajaduras, $41 \%$ pandeos y el $14 \%$ colapsos parciales. Estadísticamente, el $50 \%$ de las viviendas de este sector se encuentran en estado ruinoso o de emergencia. Sin embargo, esto no ha sido una limitación para la sobreocupación de predios, ya que es el lugar con mayor concentración de tugurios ${ }^{4}$ en Lima Metropolitana: 10000 viviendas aproximadamente (Centro de Promoción Urbana, 2009).

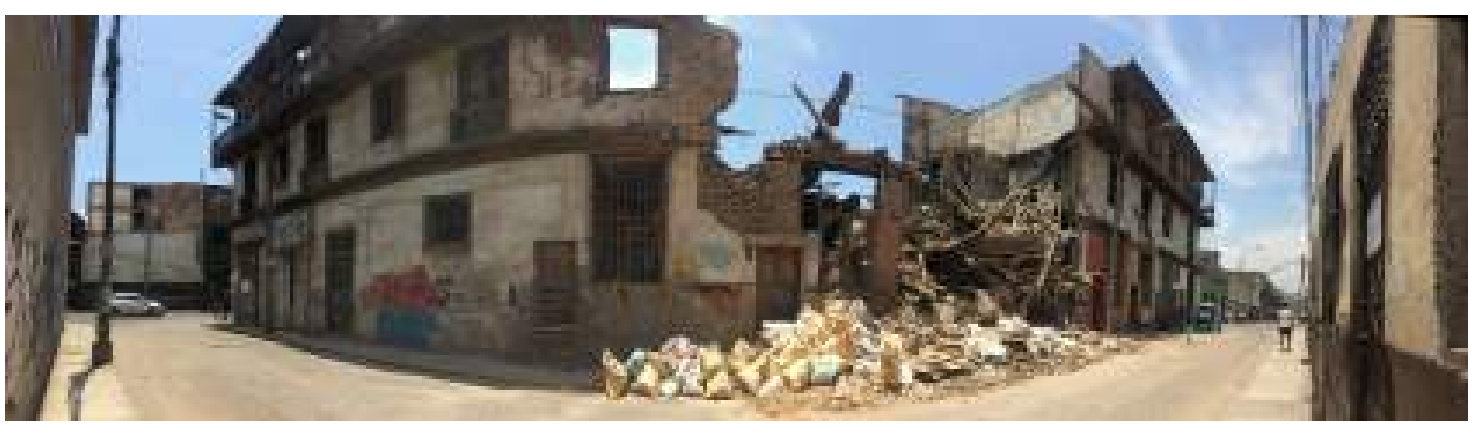

Figura 3

Edificio El Buque visto desde la calle Cangallo

Con respecto a la dotación de servicios, el 97 \% de los inmuebles en Barrios Altos cuentan con acceso a servicios de agua, desagüe y luz (Prolima, 2014). Sin embargo, el dato no contempla el estado de las instalaciones, en su mayoría colapsadas por la falta de mantenimiento. La red de alumbrado supone un constante peligro por la manipulación no regulada de la derivación multipredial a partir de una sola red, lo que genera sobrecarga;

4 Tugurio se define como vivienda precaria que no tiene las mínimas condiciones de habitabilidad, carente o deficiente en servicios de agua, desagüe y alumbrado eléctrico, así como con un precario entorno urbano (Plan Metropolitano Lima-Callao, 1980). 
asimismo, el uso de cableado no apto y su exposición a la intemperie acarrean un riesgo constante de incendio. Por otro lado, la red de agua y alcantarillado se encuentra deteriorada por la falta de mantenimiento, y aún se encuentran tuberías caducas de hierro fundido con perforaciones y óxidos naturales ocasionados por el paso del tiempo. Esto da como resultado constantes afloramientos, genera problemas a la salud y debilita la estructura del inmueble por humedad.

\section{Desarraigo social}

La falta de identidad con el emplazamiento guarda relación directa con la condición de ocupación. La encuesta realizada en el 2001 por el Instituto Nacional de Defensa Civil (Indeci) indicó que el 61 \% de las viviendas eran habitadas por inquilinos; el $31 \%$ lo eran con autorización del propietario, pero sin pago; y el 1,6\% estaban ocupadas por el propietario.

Los inquilinos, por su origen foráneo y su naturaleza nómade en el territorio, no tienen vínculos sociales que involucren su participación social y económica para el mejoramiento del sector. Su inacción ocasiona conformismo por la precariedad de sus viviendas y el desinterés en el mejoramiento de su emplazamiento, por lo que aumentan los conflictos sociales, se debilitan los mecanismos de participación ciudadana y se reducen los frentes de acción para la protección ante situaciones de emergencia (sismos, incendios, etcétera).

\section{Tráfico de terrenos}

Barrios Altos se enfrenta a la amenaza creciente de la desresidencialización, surgida a partir de la informalidad y el tráfico de terrenos residenciales. Aprovechando el actual bajo costo por metro cuadrado de los terrenos, la localización estratégica del sector y la falta de fiscalización por parte de la MML han surgido nuevas edificaciones de 10 pisos en material noble para uso de almacén, las que ocupan el lugar de antiguos inmuebles residenciales y han desplazado a las familias que las habitaban. El aumento de estas edificaciones comerciales fuera de escala dentro del sector promueve la emigración de las familias barrioaltinas y con ello su memoria urbana, cultura 
e identidad; además, debilita los mecanismos de participación ciudadana al reducirse la población originaria. Este fenómeno a largo plazo traerá consigo la especulación inmobiliaria, los desalojos forzosos y la aparición de problemáticas relacionadas con la falta de residencialidad: inseguridad ciudadana, incendios, colapsos, entre otros.

Hoy en día, decenas de familias vienen siendo afectadas, pues ante la falta de conocimiento del antecedente legal de la propiedad terminan estafadas o desalojadas por traficantes de terrenos, quienes logran hacerse de la titularidad de inmuebles habitados por posesionarios con propietario ausente bajo la figura legal de la tercera venta o adquisición de buena $\mathrm{fe}^{5}$. Así, los expulsan del sector y les quitan la posibilidad de participar en proyectos municipales de renovación urbana ${ }^{6}$.

\section{Descoordinación político-institucional}

Actualmente, la gestión de Barrios Altos demuestra una falta de claridad debido al cruce de competencias entre organismos institucionales que forman parte del Ministerio de Cultura y la Municipalidad Metropolitana de Lima: Programa Municipal de recuperación del Centro Histórico de Lima (Prolima), Empresa Inmobiliaria de Lima (Emilima) y la Gerencia de Desarrollo Urbano (GDU). Por ello, se han generado trabas para la inversión privada, ya que resulta engorroso el proceso administrativo para los predios del CHL, que cuentan con normativas especiales dirigidas a los diferentes organismos institucionales que los rigen.

Por otro lado, la falta de voluntad política para el fomento del desarrollo de Barrios Altos y la discontinuidad política que ocurre al término de cada mandato (lo cual es un indicador común en la política peruana) generan desconfianza y falta de reconocimiento en la población receptora. Los temas

5 Según el artículo 2014 del Código Civil, se adjudica la titularidad de un inmueble a un comprador a partir de la tercera venta, aunque después se identifique la ilegalidad de dicha venta, ya que se justifica que el tercero registral realizó la compra con desconocimiento de causa.

6 Según indicó el economista Miles Calean en 1950, la renovación urbana se refiere a la renovación de edificios necesarios debido a su envejecimiento o para adaptarlos a nuevos usos. 
de renovación urbana para la recuperación de tugurios con permanencia de sus habitantes presentan limitaciones administrativas debido al aspecto legal y normativo, que no se ajusta por completo a la realidad de Barrios Altos y que restringe a los residentes sin darles herramientas de gestión ni de mejoramiento para sus viviendas (Centro de Investigación, Documentación y Asesoría Poblacional, 2008).

Evidencia de la limitación político-administrativa es la desarticulación de proyectos metropolitanos que favorecen el desarrollo integral de Barrios Altos, lo cual genera estancamiento en el mejoramiento urbano de la zona. La desactivación del Plan Específico de Barrios Altos 2014-2025 privó el impulso del fortalecimiento de la identidad barrioaltina con acciones de renovación y densificación urbanas como mecanismo de revaloración cultural e inmobiliaria; además, la desactivación del proyecto Vía Parque Rímac propuesto en el Plan Metropolitano de Desarrollo Urbano (PLAM) al 2035 desvaneció la promesa de interconectar Barrios Altos con zonas aledañas, así como la generación de espacios públicos dotados con áreas verdes y equipamiento cultural que pudieran cubrir la activa demanda de estos.

\section{Esquemas económicos inadecuados}

Según lo señala el Plan Local de Seguridad Ciudadana 2015-MML, en el área del CHL el ingreso familiar promedio es de S/ 894 y el $58 \%$ de la población pertenece al nivel socioeconómico C. Estos datos significan que la población de Barrios Altos enfrenta obstáculos financieros que le impiden acceder a créditos privados para realizar mejoras en sus viviendas. Las precarias condiciones socioeconómicas, el alto índice de desempleo, los bajos ingresos económicos, el riesgo crediticio, las deudas, la falta de garante, entre otros, condenan a sus residentes a no poder acceder a recursos que mejoren sus condiciones de habitabilidad; de esta manera, el deterioro de la zona es continuo.

Paralelamente, no se emprenden programas de financiamiento gubernamentales que estén adaptados a la realidad de Barrios Altos en el contexto del CHL. Si bien actualmente se llevan adelante programas sociales de vivienda, como Mivivienda y Techo Propio, — a cargo del Ministerio de Vivienda, Construcción y Saneamiento (MVCS)—, estos se enfocan en el inmueble 
nuevo, mientras que en Barrios Altos se tiende a priorizar la renovación urbana, para lo cual no hay programas. Sumado a ello, la desactualización de la normativa de cooperativas como medio para alcanzar financiamiento ha dejado sin herramientas a las agrupaciones vecinales y ha desarticulado, a su vez, los incipientes mecanismos de participación vecinal.

El análisis del sector permitió identificar la existencia de los actores institucionales y vecinales. Las estrategias de acción que envuelven a los actores institucionales implican mecanismos de acción política, los cuales se encuentran fuera del alcance de un habitante promedio. A su vez, se ha reconocido que el actor vecinal si bien tiene grandes limitantes, con las adecuadas herramientas técnicas, financieras y de liderazgo puede hacer frente a las trampas de riesgo cotidiano. Por medio de la organización y empoderamiento vecinal se pueden ejecutar proyectos de autogestión que incluyan la recuperación progresiva del predio donde residen, de modo que se salvaguarde para el futuro su patrimonio familiar edificado.

Según el análisis de trampas de riesgo en Barrios Altos, se ha identificado que la vulnerabilidad de las edificaciones ante la condición de emergencia es de primera importancia. Intersecada con la injerencia de acción de los actores, se esboza una primera estrategia para reducir riesgos cotidianos de vulnerabilidad estructural: la ejecución de proyectos de mejoramiento de las condiciones de habitabilidad, a cargo de juntas vecinales organizadas. La visión final de estos proyectos a pequeña escala es demostrar que los residentes (propietarios, inquilinos y posesionarios) pueden ejecutar planes de mejoramiento en predios ruinosos a través de la cooperación económica, organizativa y el trabajo. Finalmente, la repetición de proyectos de este tipo de iniciativas en predios aledaños pretende promover la recuperación de Barrios Altos desde el interior de las manzanas, con miras a una escala macro que comprenda finalmente a los actores institucionales.

\section{CASO DE ESTUDIO QUINTA BASELLI: PROPUESTA MODELO PARA LA INTERRUPCIÓN DE TRAMPAS DE RIESGO COTIDIANO}

El caso de estudio ha sido desarrollado en el marco del concurso Propuestas Innovadoras para la Prevención y Reducción de Riesgos Cotidianos en 
Barrios Altos, Centro Histórico de Lima. Fue organizado por Development Planning Unit de University College London (DPU- UCL) en colaboración con las ONG locales Centro de Investigación, Documentación y Asesoría Poblacional (Cidap), Centro de Desarrollo Urbano (Cenca) y Foro Ciudades Para la Vida. Resultó ganador el Programa Modelo de Mínima Intervención Autosostenible: Quinta Baselli, y se otorgó el financiamiento para el desarrollo de la primera etapa del proyecto. Este se encuentra a cargo del Colectivo Más Ciudad, conformado por los bachilleres en Arquitectura Vanesa Olazábal Mendoza y Martín Paz Olaechea, con la participación del dirigente vecinal Luis Gonzales y la Junta Vecinal de la Quinta Baselli.

El programa mencionado, ejecutado en la Quinta Baselli como proyecto piloto, se propone como un mecanismo de mejoramiento de viviendas denominadas quintas ${ }^{7}$, ya que estas tienen presencia mayoritaria en el CHL: representan el $80 \%$ del total edificado, según datos recogidos por Indeci en 2001. El proyecto consiste en lograr la recuperación de inmuebles con potencial turístico, mediante estrategias de autoconstrucción y autofinanciamiento logradas por la acción participativa de los habitantes de las quintas. Los fines son repetir el proyecto piloto en quintas aledañas.

\section{Respecto de la Quinta Baselli}

La Quinta Baselli, predio base del programa modelo propuesto, fue edificada en 1929 y se ubica en Jr. Junín 1342, Barrios Altos. La quinta cuenta con 72 unidades inmobiliarias, de las cuales dos son de uso comercial y se localizan en el frontis del predio, mientras que 70 son de uso residencial y se encuentran en el interior. La quinta comprende seis unidades deshabitadas, de las cuales dos se encuentran derruidas por colapso desde el 2002 y cuatro por la ausencia de sus propietarios ${ }^{8}$. Arquitectónicamente, el recinto de 13 metros de fachada por 100 metros de fondo está conformado por dos

7 Según la Norma G.040 del Reglamento Nacional de Edificaciones, se define como quinta al conjunto de pequeños departamentos edificados sobre un lote multifamiliar, con acceso por un espacio común directamente desde la vía pública y que en algunos casos poseen servicios domiciliarios.

8 Información que recogí en noviembre del 2016. 
niveles: el primer nivel está construido con adobe y el segundo con quincha. Dispone de dos accesos verticales fabricados de madera, el primero de estos tenía acabado de mármol de Carrara, pero desapareció durante la década de 1980. Espacialmente, el recinto dispone de un solo ingreso principal por el cual se accede a las unidades de vivienda ubicadas a lo largo de tres patios centrales de uso común, que evidencian su colorido gracias al acabado en baldosa empastada, de la que solo quedan rezagos. En un inicio, la quinta contó con servicios básicos de agua y desagüe de uso común, y se destinó la batería de servicios higiénicos al fondo del inmueble. Hoy está en desuso, ya que los predios cuentan con instalaciones de servicios de luz, agua y desagüe independientes. En términos sociales, la Quinta Baselli está compuesta por $20 \%$ de propietarios, $30 \%$ de posesionarios y $50 \%$ de inquilinos. Según la World Monuments Fund, desde el 2008 la Quinta Baselli ha sido catalogada como uno de los 100 inmuebles en riesgo del CHL debido al deterioro y la falta de mantenimiento de sus instalaciones.
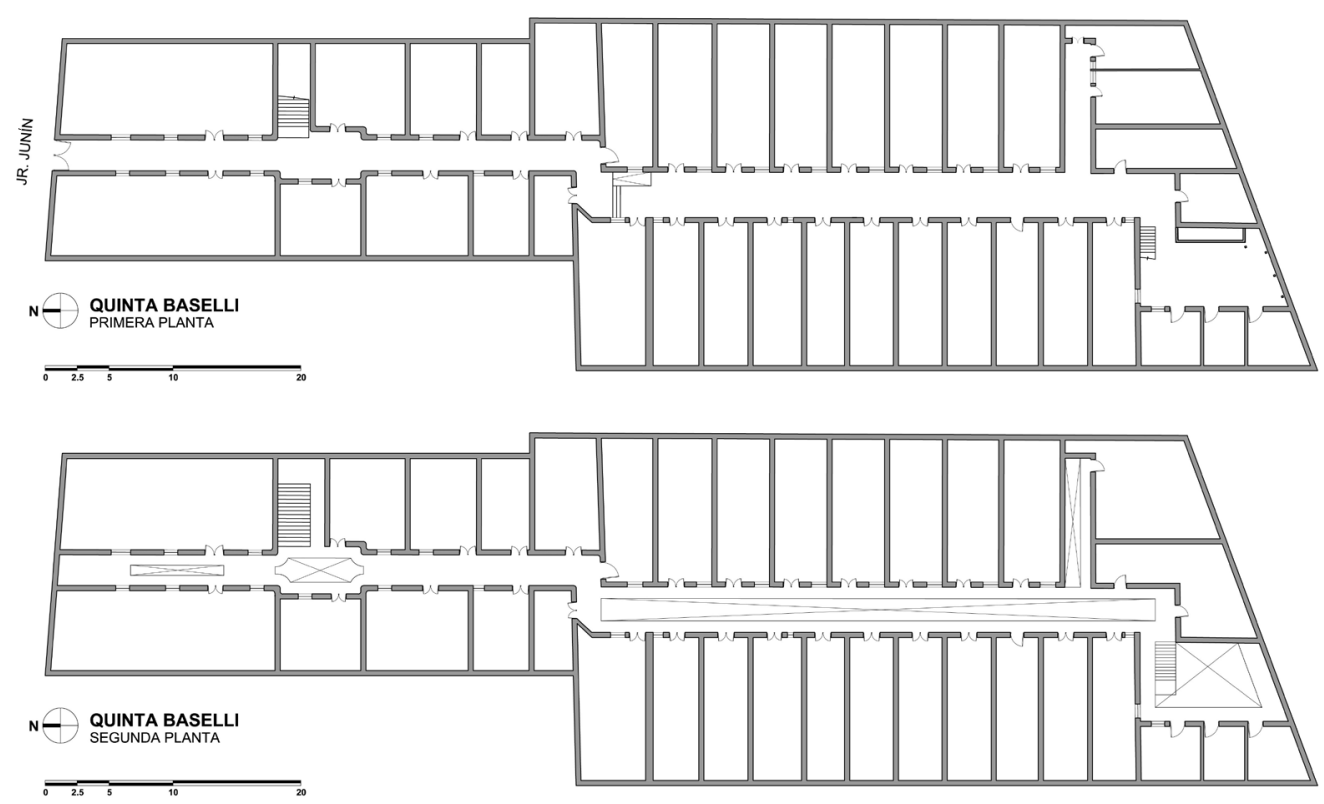

Figura 4

Planta arquitectónica de la Quinta Baselli

Elaboración: Iván Rojas Villafuerte 


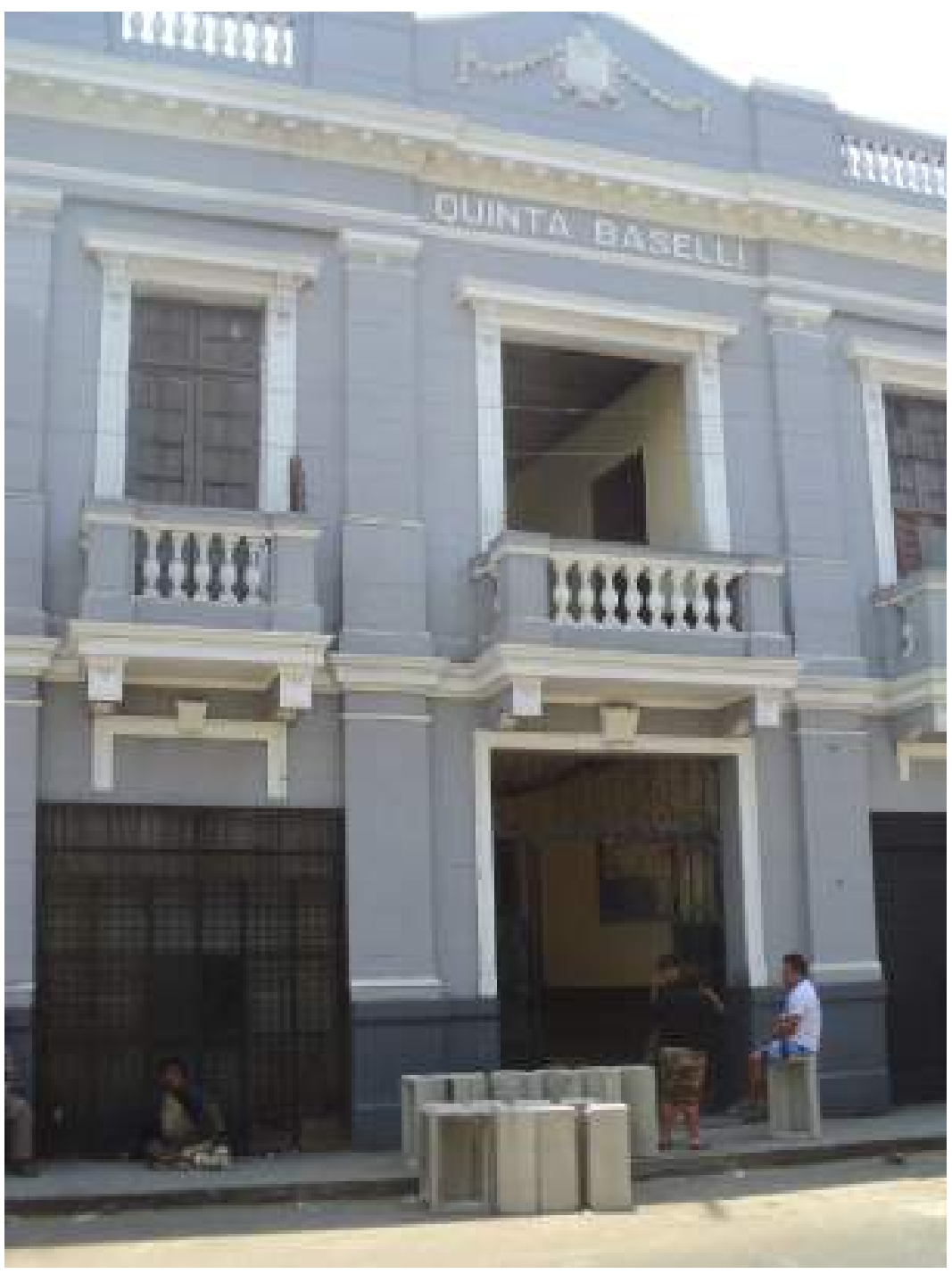

Figura 5

Fachada de la Quinta Baselli 


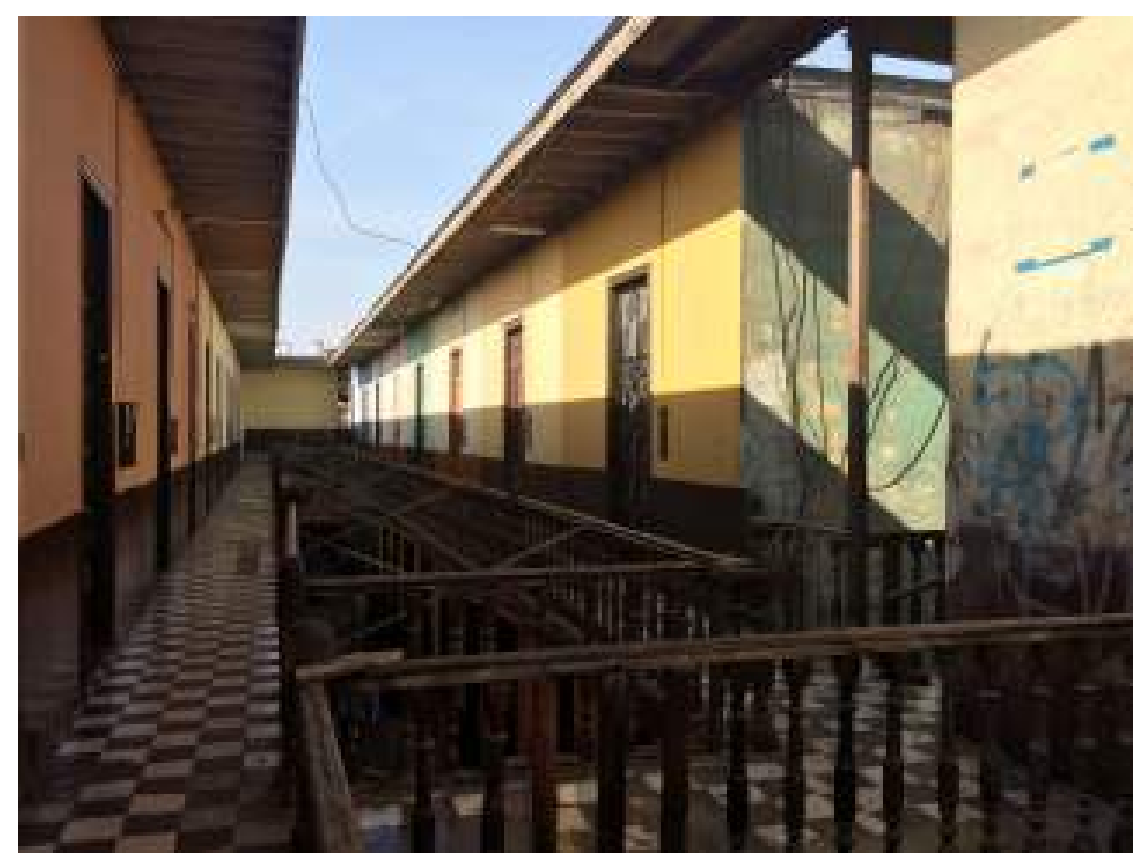

Figura 6

Segundo nivel de la Quinta Baselli

Se escogió la Quinta Baselli como modelo de intervención por reunir gran parte de la problemática residencial de Barrios altos, como poseer la denominación de Inmueble de Valor Histórico ${ }^{9}$, padecer de debilitamiento estructural, servicios básicos deficientes (especialmente colapso del servicio de alcantarillado) y sistema constructivo republicano en mal estado debido a la precaria intervención de sus residentes. Socialmente, la Quinta Baselli está representada por una joven organización vecinal que actualmente lucha contra la inseguridad ciudadana, drogas, etcétera, y que cuenta con la

9 Según la Norma A.140 del Reglamento Nacional de Edificaciones, un inmueble de valor monumental es aquel que sin haber sido declarado monumento reviste valor arquitectónico 0 histórico declarados expresamente por el Ministerio de Cultura. 
participación activa de solo el $20 \%$ del total de residentes, debido a la poca injerencia de la población restante y por conflictos entre vecinos.

\section{Modelo de autogestión}

La estrategia del Programa Modelo de Mínima Intervención Autosostenible: Quinta Baselli se planteó como mecanismo de interrupción de trampas de riesgo cotidiano y constaba de dos planes de acción a corto y largo plazo, de los cuales el beneficiario directo sería el actor vecinal. Por ello, ambas etapas se regirían sobre la base de una organización vecinal en quintas, con el objetivo de fortalecerla hasta el final del proceso al tratar el predio como unidad y alejarlo de los conflictos de intereses debido a las subdivisiones que se presentasen.

A corto plazo se propuso una primera etapa, también denominada impulsora, en la cual se mejorarían las condiciones de habitabilidad de la quinta mediante la dotación de servicios básicos (luz, agua o desagüe) que permitieran reducir las amenazas de riesgos cotidianos relacionados con el colapso estructural. Económicamente, esta etapa sería promovida por un organismo donante que facilitaría el acceso a financiamiento para realizar acciones en pro del mejoramiento de las viviendas.

La segunda etapa fue denominada autosostenible. En ella se propuso llevar a cabo acciones a largo plazo en las que se garantizase el mantenimiento del predio a través de la remodelación o habilitación de dos unidades de vivienda deshabitadas, con el propósito de ser rentadas para la generación de utilidades. El financiamiento se planteó como inversión privada a modo de concesión tripartita: inversionista, propietario y quinta. Con la incorporación de la quinta al modelo de concesión, se pretende garantizar ingresos económicos para el mantenimiento del predio.

\section{Aplicación del modelo en la Quinta Baselli}

La ejecución del programa piloto en Quinta Baselli comenzó en noviembre del 2016 y se llevó a cabo durante dos meses y medio, pero solo se efectuó la primera etapa por poca disponibilidad de presupuesto. Específicamente, la primera etapa del modelo descrito anteriormente consistió en la solución del 
servicio de alcantarillado, ya que era fuente de debilitamiento por humedad de la base de las estructuras de abobe del primer nivel. Esto ponía en riesgo la capacidad portante del edificio, así como la salud de los residentes a raíz de los recurrentes afloramientos de aguas servidas.

En la esta etapa se cambió el sistema de alcantarillado de la red principal de la quinta, ubicado en el área común del primer nivel; además, se contempló el reemplazo de las 14 cajas de registro y de las tuberías de hierro originales (de la construcción de 1929) por tuberías de PVC de 6 pulgadas. Espacialmente, se incorporaron materiales compatibles con el contexto, como la renovación de las tapas de desagüe de fierro y la conservación del colorido piso de baldosas empastadas. Para esto último, se rescataron la mayor cantidad posible de piezas originales, y se reincorporaron y expusieron en el primer patio; el resto de la intervención consistió en un acabado de baldosas empastadas en tonalidad salmón, que es el color predominante en el piso.

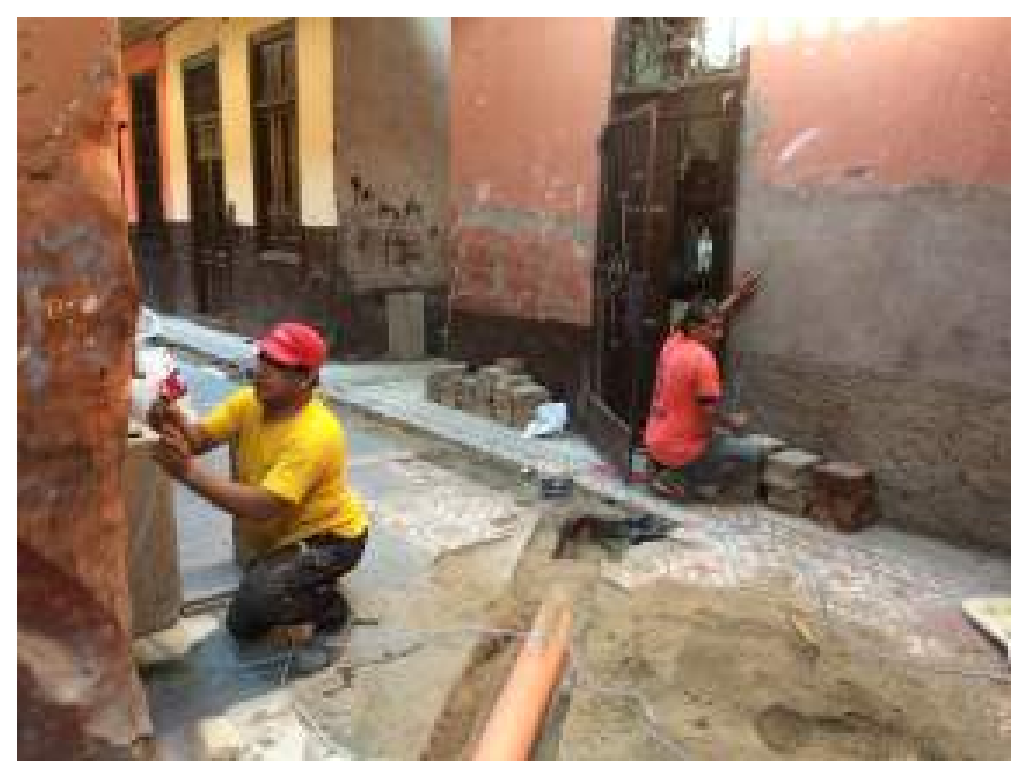

Figura 7

Trabajos durante la ejecución del proyecto piloto en la Quinta Baselli 


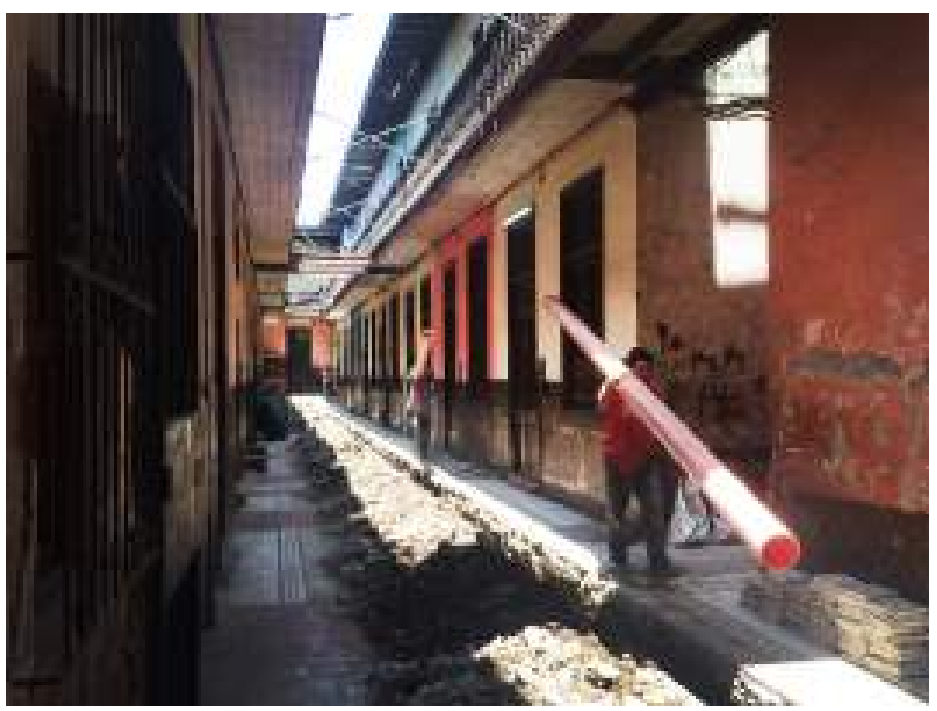

Figura 8

Acarreo de materiales durante la ejecución del proyecto piloto en la Quinta Baselli

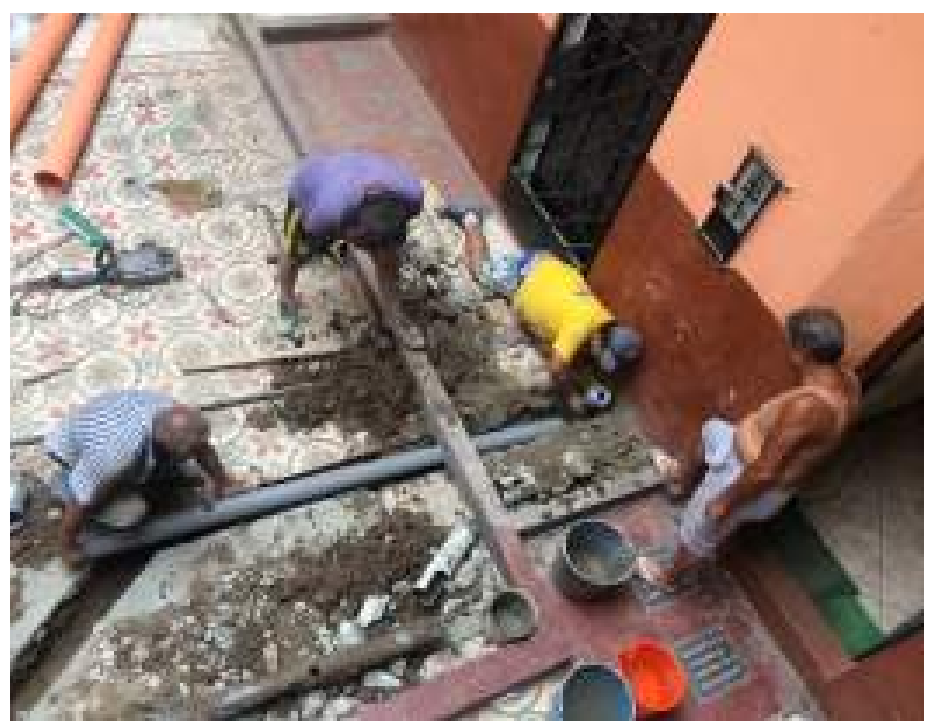

Figura 9

Instalación de tubería de PVC en la troncal del sistema de desagüe de la Quinta Baselli 


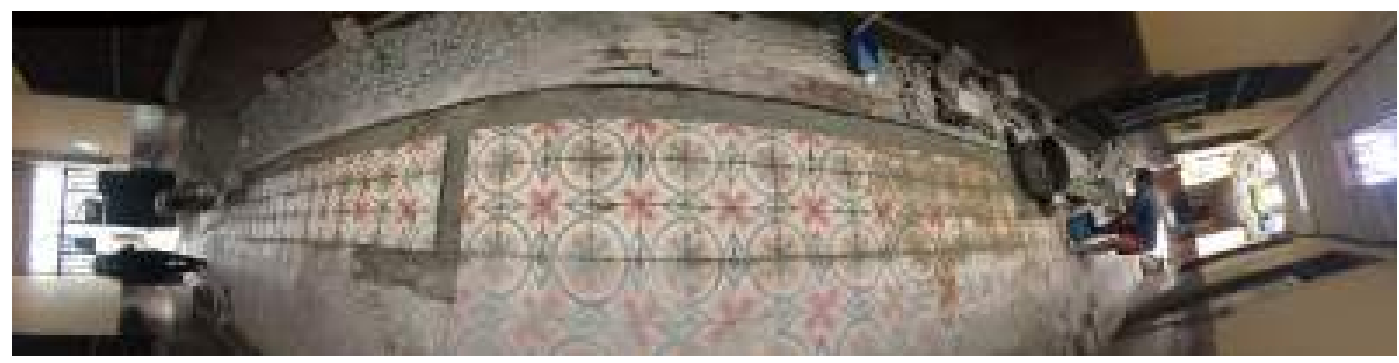

Figura 10

Reintegración de baldosas empastadas en el ingreso de la Quinta Baselli

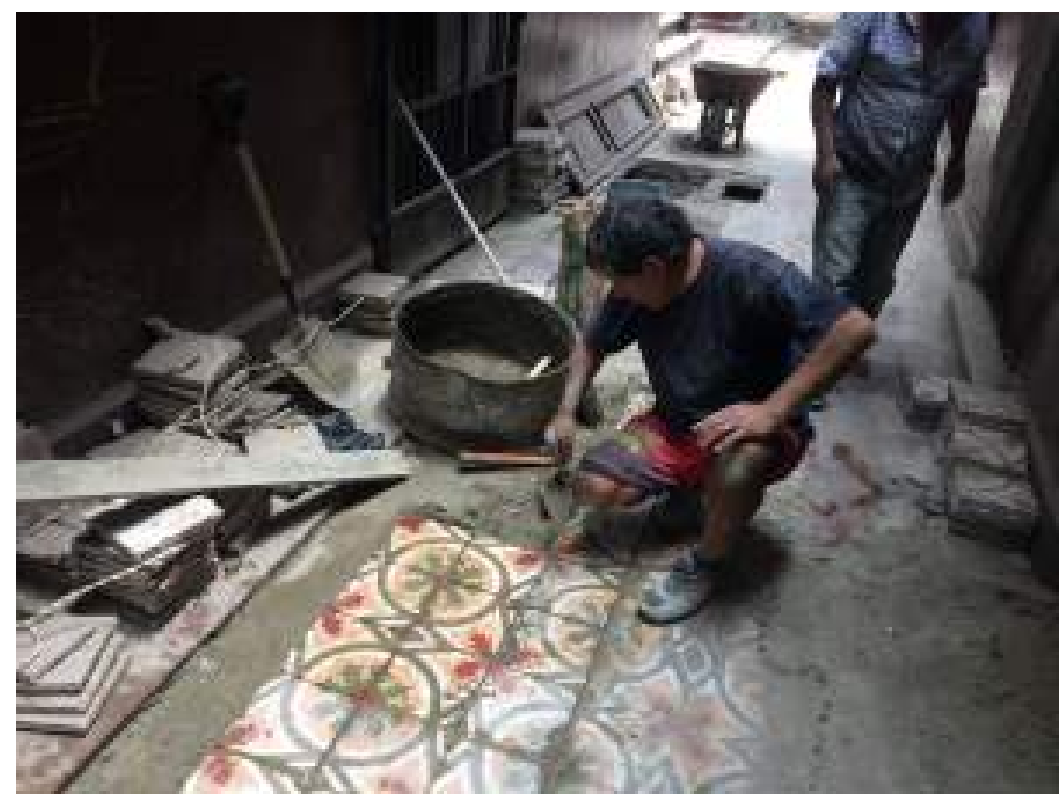

Figura 11

Instalación de baldosas empastadas originales 
En cuanto a la participación ciudadana, la junta vecinal tuvo como principal tarea habilitar mecanismos de diálogo y consenso entre el equipo técnico, los financiadores, las organizaciones externas y los residentes. A su vez, debido a que el proyecto no contempló la autoconstrucción, se llegó al consenso de que la mano de obra técnica estuviera conformada por obreros de manzanas aledañas con experiencia en trabajos de construcción, por lo que la junta vecinal, por medio de comisiones de trabajo, asumió la responsabilidad de control y seguimiento de la obra.

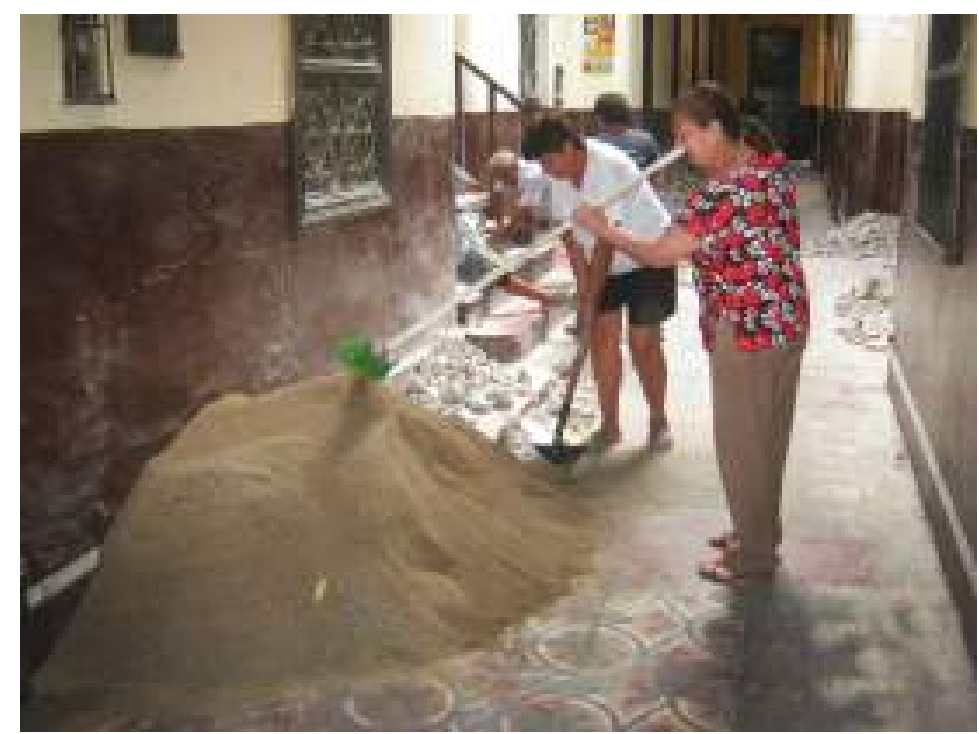

Figura 12

Participación de los vecinos de la Quinta Baselli en actividades de orden y limpieza de agregados de construcción 


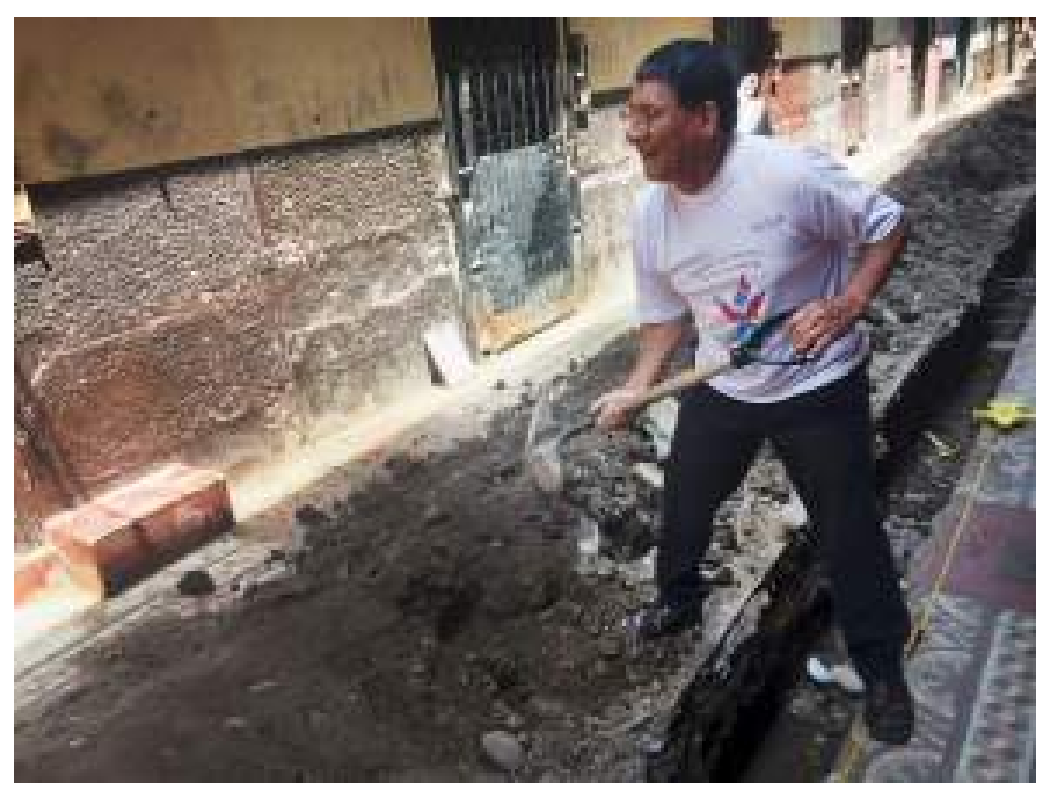

Figura 13

Participación de los vecinos de la Quinta Baselli en eliminación del desmonte

\section{Resultados}

a) Componente infraestructura

Si bien el colapso del alcantarillado ha sido un problema evidente por los atoros y afloraciones, se halló que el colapso del sistema de agua potable era aún más alarmante. Durante la ejecución del proyecto, se evidenciaron filtraciones recurrentes de agua potable que generaban la pérdida de hasta 30 litros de agua al día; además, mediante visitas de campo, se comprobó que tal situación se repetía en predios aledaños. Ante ello, se infiere que el debilitamiento estructural del sector de Barrios Altos se debe a la exposición a la humedad generada por la manipulación informal de las conexiones condominiales para acceder al agua potable. 


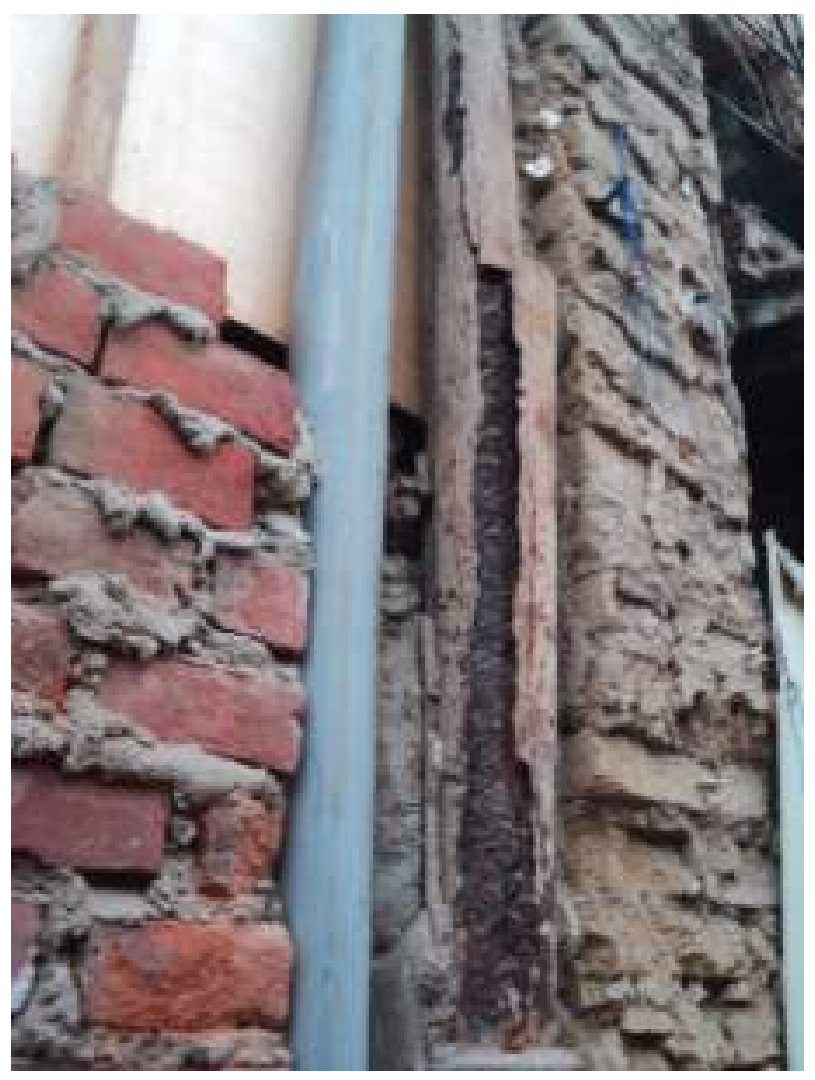

Figura 14

Tuberías de desagüe de PVC y de hierro en la Quinta Baselli 


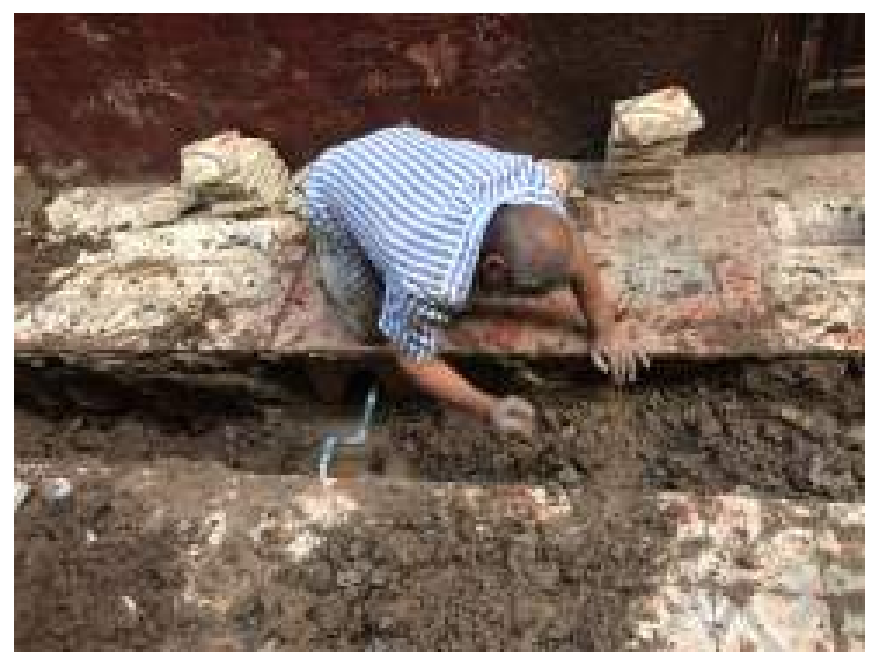

Figura 15

Evidencia de pérdida de agua potable por filtraciones

\section{b) Componente político institucional}

Durante el proceso, la junta de vecinos de la Quinta Baselli intentó establecer un diálogo con la MML, con fines de informar a este organismo del proyecto piloto y buscar su participación. Como respuesta se recibió la colaboración ausente, es decir, el proyecto no obtuvo trabas por normativa o multas; sin embargo, no se hizo el seguimiento por parte de sus funcionarios, lo que limitaría los alcances y la promoción de proyectos de este tipo en la zona.

\section{c) Componente social}

La organización vecinal en la Quinta Baselli resultó vital para gestionar el diálogo entre los residentes y los actores externos (técnicos, organizaciones, etcétera). La organización vecinal evidenció formalidad y cooperación, sin las cuales no se hubiera podido acceder a asesoría técnica de organizaciones no lucrativas (Colectivo Más Ciudad) ni financiamiento de cooperación internacional (Proyecto cLima Sin Riesgo). A su vez, estas características le permiten seguir presentándose ante instituciones, universidades, inversores 
privados y público en general que tengan interés en la problemática de Barrios Altos. Una primera prueba de ello fue la invitación que recibieron al evento internacional por el Climate, Development, Knowledgement \& Network (CDKN) en Ecuador, en el marco del ONU - Hábitat III, donde expusieron los alcances e impacto del proyecto. Por otro lado, se promovió la actividad voluntaria local con la realización del primer voluntariado técnico, dirigido a estudiantes de Arquitectura de pregrado.

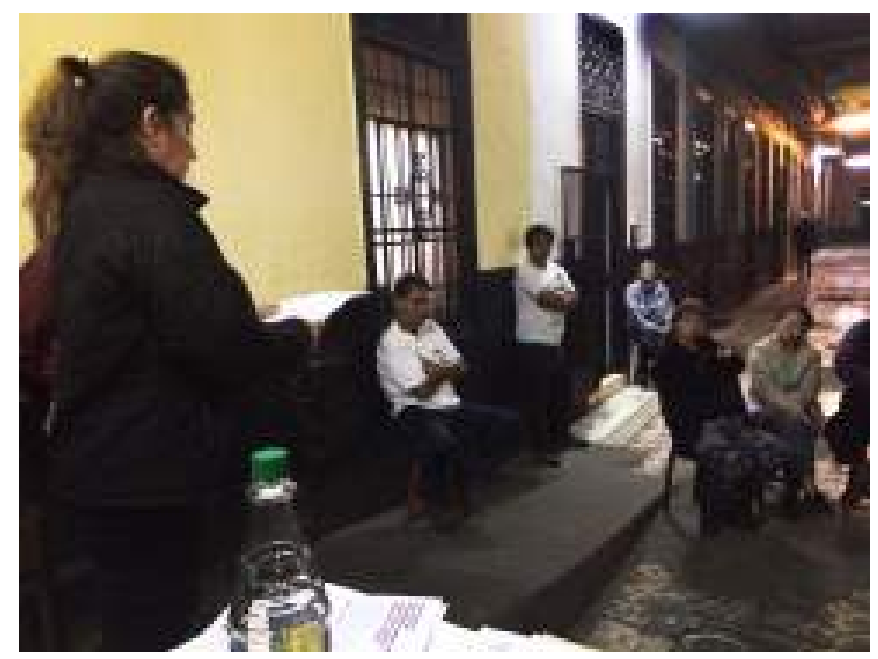

Figura 16

Junta de vecinos de la Quinta Baselli. Ada Rendón expone sus experiencias durante su presentación en Ecuador como representante de la quinta

Por otro lado, el acompañamiento de la junta vecinal de Quinta Baselli permitió el manejo de conflictos recurrentes dentro de un predio subdividido, generalmente entre vecinos del primer y del segundo piso, y consensuar las necesidades internas de los residentes para proyectarlas al equipo técnico. Con respecto a las limitaciones por la condición del predio, se rescata la estrategia acordada con la mayoría inquilina, en la que se plantea un mecanismo de participación económica: $50 \%$ del dueño y $50 \%$ del inquilino. 


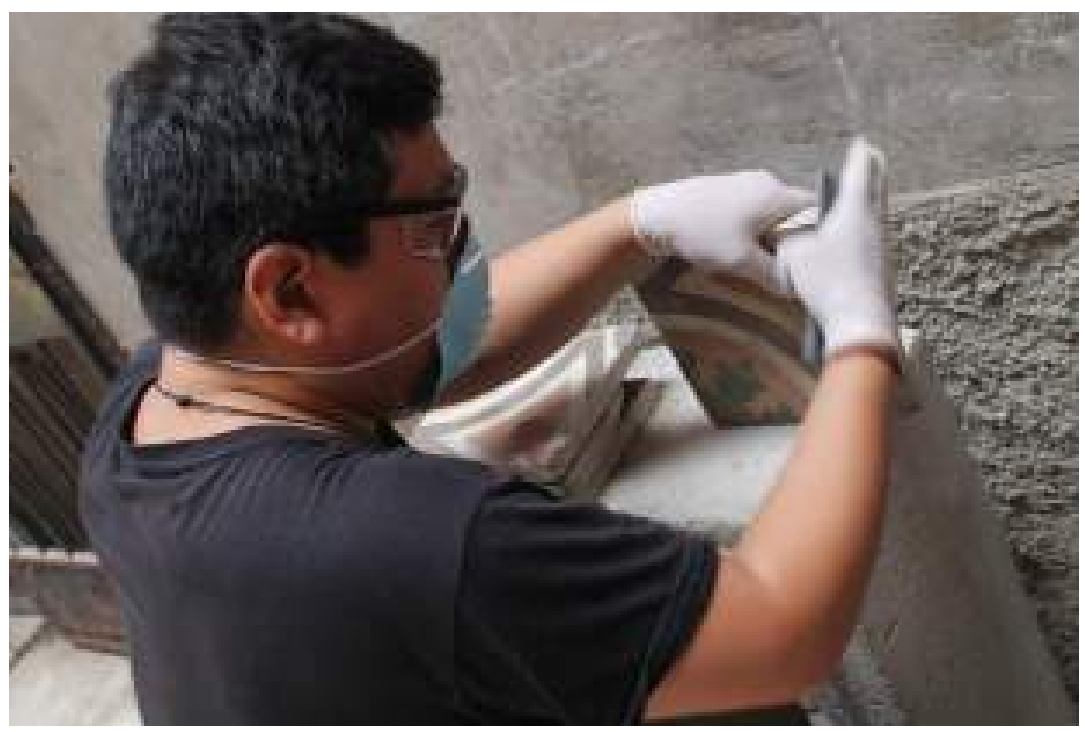

Figura 17

Voluntarios en trabajos de limpieza de las baldosas empastadas originales

\section{CONCLUSIONES}

Espacialmente, Barrios Altos presenta una ubicación céntrica para acceder a servicios metropolitanos, sin embargo, la marginación de la zona en temas urbanos le resta a su desarrollo integral e incrementa progresivamente las trampas de riesgo cotidiano. Esto se debe, principalmente, a que los actores institucionales no demuestran interés en mejorar el sector, lo cual se evidencia en la ausencia de programas de recuperación de infraestructura y en programas sociales de financiamiento.

Ante la negativa institucional, la organización vecinal resulta importante como estrategia para el mejoramiento de las condiciones de habitabilidad. Sin embargo, cabe notar que la organización vecinal en Barrios Altos resulta complicada por el flujo constante de inquilinos y la falta de responsabilidad de sus miembros. 
El caso de estudio Quinta Baselli se enfoca en la consolidación participativa del actor vecinal dentro de las estrategias de acción para interrumpir trampas de riesgo cotidiano. Actualmente, se cuenta con el $20 \%$ de la participación vecinal, por lo que se espera la consolidación de sus mecanismos de participación para así promover la ejecución de la segunda etapa del proyecto.

Finalmente, se debe señalar que la iniciativa vecinal, como se observa en la intervención de la Quinta Baselli, debe ser tomada como una estrategia inicial, ya que el mejoramiento del sector debe incluir la cooperación de todos los actores con injerencia en Barrios Altos, para así garantizar la repetición y sostenibilidad de las iniciativas.

\section{REFERENCIAS}

Carta de Veracruz (22 de mayo de 1992). Criterios para una política de actuación en los centros históricos de Iberoamérica. Recuperado de sitio de internet de la Municipalidad de Lima: http://www.munlima. gob.pe/images/descargas/programas/prolima/compendio-patrimoniointernacional/1992-Carta-Veracruz.pdf

Centro de Investigación y Asesoría Poblacional. (2008). Acciones de Renovación Urbana para hacer de Lima un centro vivo. Recuperado de http://www.cidap.org.pe/img_upload/04fbbea1283917eab076d5de 478c9f2b/informe_aniversario_de_Lima.pdf

Centro de Promoción Urbana (2008). Ley de Renovación Urbana. Recuperado de http://www.cepromur.org/introduccion.html\#LeyRU

De-los-Ríos, S., De-la-Serna, J., Chávez Marroquín, J. L., Barbacci, N., y Córdova, R. (2011). Centro Histórico de Lima, patrimonio humano y cultural en riesgo. Lima: Centro de Investigación, Documentación y Asesoría Poblacional.

Harms, H., y Ludeña, W. (1996). Vivir en el "centro". Vivienda e inquilinato en los barrios céntricos de las metrópolis de América Latina. Hamburgo: Technische Universitat Hamburg-Harbur. 
Instituto Metropolitano de Planificación y Municipalidad Metropolitana de Lima. (1999). Proyecto de Renovación Urbana en Barrios Altos. Lima.

Instituto Nacional de Defensa Civil. (2001). Análisis de la vulnerabilidad y determinación del riesgo I etapa - Lima Cercado. Lima.

Municipalidad Metropolitana de Lima. (2014). Plan específico de Barrios Altos (Cercado de Lima y El Agustino) 2014-2025. Resumen ejecutivo. Recuperado de sitio de internet de la Municipalidad de Lima: http:// www.munlima.gob.pe/images/descargas/gerencias/GDU/PlanEspecifico-Barrios-Altos-Resumen-Ejecutivo.pdf

Municipalidad Metropolitana de Lima. (2015). Plan Distrital de Seguridad Ciudadana del Cercado de Lima. Lima.

Programa Municipal para la Recuperación del Centro Histórico de Lima. (2014). Plan Maestro del Centro Histórico de Lima al 2025. Recuperado de sitio de internet de la Municipalidad de Lima: http://www.munlima. gob.pe/images/descargas/programas/prolima/PLAN-MAESTRO.pdf

Reyes, A. (2004). Historia urbana de Lima: Los Barrios Altos 1820-1880. Investigaciones Sociales, 8(13), 135-162.

Riesgo Urbano en busca de nuevas miradas. (junio del 2015).cLima sin Riesgo, (1). Recuperado de https://cdkn.org/wp-content/uploads/2015/07/ CSR_Newsletter-No-1_Spanish_WEB.pdf

Shimabukuro, A. (2015). Barrios Altos: Caracterización de un conjunto de barrios tradicionales en el marco del Centro Histórico de Lima. Revista de Arquitectura, 17(1). Bogotá: Fondo Editorial Universidad Católica de Colombia. 